\title{
Site-Specific Connexin Phosphorylation Is Associated with Reduced Heterocellular Communication between Smooth Muscle and Endothelium
}

\author{
Adam C. Straub ${ }^{a}$ Scott R. Johnstone ${ }^{a}$ Katherine R. Heberlein ${ }^{a, b}$ \\ Michael J. Rizzo ${ }^{a} \quad$ Angela K. Best ${ }^{a} \quad$ Scott Boitano $^{c}$ Brant E. Isakson ${ }^{a}$ b \\ ${ }^{a}$ Robert M. Berne Cardiovascular Research Center, and ${ }^{b}$ Department of Molecular Physiology and Biological Physics, \\ University of Virginia, Charlottesville, Va., 'Department of Physiology, University of Arizona, Tucson, Ariz., USA
}

\section{Key Words}

Myoendothelial junction - Gap junction - Phosphorylation • Heterocellular communication - Endothelium · Smooth muscle

\begin{abstract}
Background/Aims: Myoendothelial junctions (MEJs) represent a specialized signaling domain between vascular smooth muscle cells (VSMC) and endothelial cells (EC). The functional consequences of phosphorylation state of the connexins (Cx) at the MEJ have not been explored. Methods/ Results: Application of adenosine 3',5'-cyclic monophosphate sodium ( $\mathrm{pCPT}$ ) to mouse cremasteric arterioles reduces the detection of connexin 43 (Cx43) phosphorylated at its carboxyl terminal serine 368 site (S368) at the MEJ in vivo. After single-cell microinjection of a VSMC in mouse cremaster arterioles, only in the presence of pCPT was dye transfer to EC observed. We used a vascular cell co-culture (VCCC) and applied the phorbol ester 12-O-tetradecanoylphorbol 13-acetate (PMA) or fibroblast growth factor-2 (FGF-2) to induce phosphorylation of Cx43 S368. This phosphorylation event was associated with a significant reduction in dye transfer and calcium communication. Using a novel method to monitor increases in intracellular calcium across the in vi-
\end{abstract}

tro MEJ, we noted that PMA and FGF-2 both inhibited movement of inositol 1,4,5-triphosphate $\left(\mathrm{IP}_{3}\right)$, but to a lesser extent $\mathrm{Ca}^{2+}$. Conclusion: These data indicate that site-specific connexin phosphorylation at the MEJ can potentially regulate the movement of solutes between EC and VSMC in the vessel wall.

Copyright $\odot 2009$ S. Karger AG, Basel

\section{Introduction}

Heterocellular signaling between endothelial cells (EC) and vascular smooth muscle cells (VSMC) in the vasculature is hypothesized to be conducted, in part, through direct cell-cell contact at myoendothelial junctions (MEJs) [for reviews, see 1-3]. The MEJ is a cellular extension from either EC or VSMC that projects through the internal elastic lamina making contact with the neighboring cell type [4]. MEJs have been shown to be highly organized with developing evidence to support the concept that they are unique signaling microdomains [for review, see 3]. Therefore, these structures are likely to coordinate a number of physiological functions.

At the MEJ, gap junctions (dodecameric channels that link cytoplasms of 2 cells together) can form heterocel-

\section{KARGER}

๑ 2009 S. Karger AG, Basel

Fax +4161306 1234 E-Mail karger@karger.ch www.karger.com www.karger.com/jvr
Dr. Brant E. Isakson

Robert M. Berne Cardiovascular Research Center

University of Virginia

PO Box 801394, Charlottesville, VA 22908 (USA)

Tel. +1 434924 2093, Fax +1 434924 6899, E-Mail bei6n@virginia.edu 
lular channels between EC and VSMC [5-7]. Connexins (Cxs) are the proteins that compose gap junctions, and Cx37, 40, 43 and 45 have been identified in the vasculature with all but $\mathrm{Cx} 45$ identified at the MEJ [8-10]. Each of these $\mathrm{Cx}$ isoforms contains multiple phosphorylation sites, and because phosphorylation of $\mathrm{Cx}$ have been shown to regulate the opening or closing gap junction channels $[11,12]$, the phosphorylation state of Cxs at the MEJ may be a crucial component in the regulation of heterocellular signaling.

There is emerging evidence that differential vascular beds exhibit different degrees of gap junction coupling at the MEJ. For example, dye transfer studies in rat mesenteric vascular beds and hamster cheek pouches demonstrate extensive heterocellular coupling at the $\operatorname{MEJ}[5,13]$, while in mouse cremasteric arterioles, a lack of dye and electrical coupling between the EC and VSMC suggested poor or nonexistent heterocellular coupling [14]. The latter observation is suggestive of (1) a lack of MEJs in the mouse cremaster bed, (2) a lack of gap junctions at the MEJ or (3) a differential regulation of gap junctions, possibly via an altered phosphorylation state of $\mathrm{Cx}$ isoforms that make up the junctions. Myoendothelial junctions have been identified in mouse cremaster arterioles, and we have recently published evidence of $\mathrm{Cx}$ expression on actin bridges (presumed to be MEJs) in mouse cremaster arterioles $[3,7,8]$. Interestingly, we also reported that the serine 368 (S368) site of Cx43 was extensively phosphorylated on the actin bridges in mouse cremaster arterioles, but not in the rat mesentery [8]. Phosphorylation at this residue reduces Cx43-associated gap junction conductance from approximately 100 to 50 picosiemens, and this regulation can be enhanced using phorbol esters that increase protein kinase $\mathrm{C}$ (PKC) activity [15]. This prompted us to test the hypothesis that phosphorylation at the S368 site provides a unique regulatory mechanism for heterocellular transfer of dye and second messengers at gap junctions residing at the $\mathrm{MEJ}$ in mouse cremaster arterioles.

Here we present for the first time that connexin phosphorylation can contribute to heterocellular communication at the MEJ. We provide evidence that the cAMP analog adenosine $3^{\prime}, 5^{\prime}$-cyclic monophosphate sodium (pCPT) reduces detection of S368 at Cx43 in mouse cremaster arterioles and promotes dye transfer to $\mathrm{EC}$ in vivo. Using the vascular cell co-culture (VCCC) model, induction of PKC activity using fibroblast growth factor-2 (FGF-2) or 12-O-tetradecanoylphorbol 13-acetate (PMA) promoted S368 phosphorylation which significantly limited dye transfer, inositol tri-phosphate $\left(\mathrm{IP}_{3}\right)$ signaling, and to a lesser extent movement of $\mathrm{Ca}^{2+}$ ions. When tak- en together, we believe this data provides correlative evidence by which heterocellular communication at the MEJ could be regulated.

\section{Methods}

\section{Animals}

Male C57/Bl6 mice (Taconic) were between 6 and 10 weeks of age and were used according to the University of Virginia Animal Care and Use Committee guidelines. For isolation of cremasteric cells, mice were euthanized with an intraperitoneal injection of $60-90 \mathrm{mg} / \mathrm{kg}$ pentobarbital. For in vivo cremasteric dye transfer experiments, mice were given up to $30 \mathrm{mg} / \mathrm{kg}$ pentobarbital prior to cremaster excision and dye injection.

\section{Vascular Cell Co-Culture}

Isolation and primary cultures of cremasteric EC and VSMC utilizing magnetic beads towards CD31 and NG2, respectively, and creation of the cremasteric VCCC, was performed as previously described $[16,17]$. Briefly, 75,000 cremasteric VSMCs were plated on one side of the Transwell for 3 days. Following the 3 days, 360,000 ECs were plated on the opposite side and incubated for an additional 3 days. In these experiments VSMCs were plated on the top of the Transwell, and EC were plated on the bottom of the Transwell. This type of cellular arrangement has not been shown to alter connexin protein localization or expression [16].

\section{Antibodies}

The specificity of total Cx43 (Cx43-T; Sigma) $[18,19]$ and Cx43 S368 (S368; Cell Signaling Technologies) [8] were previously described.

\section{Immunocytochemistry}

Visualization of proteins on the VCCC was performed as described [17]. Briefly, VCCC Transwells were frozen sectioned transverse to the Transwell, blocked in $10 \%$ fish skin gelatin, $0.25 \%$ Triton X-100, 0.5\% BSA in PBS, and imaged on an Olympus Fluoview confocal microscope.

Quantification of antibodies on the actin bridge between EC and VSMC was performed as previously described [8]. Briefly, using an Olympus Fluoview confocal microscope, whole-mount arterioles were visualized using phalloidin-conjugated Alexa594, and focal planes with perpendicular EC and VSMC were identified. Individual line scans across the actin bridges were used to determine pixel intensity of the primary antibody as it related to continuous phalloidin intensity across the internal elastic lamina.

\section{Immunogold Labeling for Electron Microscopy}

Immunolabeling was performed as previously described in [17]. Briefly, tissues were fixed with $4 \%$ paraformaldehyde $/ 0.5 \%$ glutaraldehyde and embedded in lowcril white. Tissue was sectioned and immunolabeled for S368 and imaged on a transmission electron microscope (Joel 1230).

\section{Chemicals}

The following chemicals were all obtained from Sigma: PMA or TPA; phorbol ester that activates PKC [20]; pCPT; membrane- 
permeable cAMP analog $(10 \mu \mathrm{M})$ [21]; FGF-2 (100 nM); rhodamine-dextran $(\mathrm{MW}=10,000 \mathrm{Da})$; carboxyfluoresceine $(\mathrm{MW}=$ 457).

\section{Dye Transfer}

Biocytin (Invitrogen) movement from cremasteric VSMC to EC on the VCCC was performed and quantified as previously described [16]. Briefly, using a pinocytotic kit (Invitrogen), VSMC were exposed to 10 -min hypertonic media with biocytin $(1.5 \mathrm{mg} /$ $\mathrm{ml}$ ) at $37^{\circ} \mathrm{C}$, followed by a 2 -min exposure to hypotonic media, and finally $10 \mathrm{~min}$ of isotonic media at $37^{\circ} \mathrm{C}$. Application of 18 $\alpha$-glycyrrhetinic acid (18 GA) to both sides of the VCCC occurred 20 min before and during loading of cells with biocytin. The biocytin-loaded VCCC were then fixed with $4 \%$ PFA, sectioned transverse to the Transwell, and analyzed using confocal microscopy [16].

For cremaster arteriole VSMC microinjections, the mouse was placed on a $37^{\circ} \mathrm{C}$ warming pad and cremaster muscles were pinned down on Sylgard-coated polyester Petri dishes and perfused with MOPS buffer at $35^{\circ} \mathrm{C}$. The first arteries supplied by feed arteries were identified as previously described [22, 23]. A micromanipulator (Eppendorf) was positioned over the cremaster muscle and a microinjector (pulse pressure $=240 \mathrm{hPa}$; Eppendorf) linked to a glass pipette (Femtotop II, tip diameter approximately $0.2-0.5 \mu \mathrm{m}$; Eppendorf) was used to inject a single VSMC from first-order arterioles with both rhodamine-dextran to mark the cell and carboxyfluoresceine to monitor small molecule movement between cells with open gap junctions. There was a 10-min delay between microinjection of a VSMC and observation of dye movement. To observe dye in VSMC and/or EC, 2 different focal planes (as indicated in fig. 2) were used. Both carbonoxolone (gap junctional communication blocker; $50 \mu \mathrm{M}$; Sigma) and pCPT (10 $\mu \mathrm{M}$ ) were topically applied to mouse cremasters for $30 \mathrm{~min}$ in the buffer before experiments were performed. If the fluorescent intensity was greater than $50 \%$ background fluorescence in the focal plane directly underneath the microinjected VSMC, this was counted as dye transfer to EC.

\section{Calcium Communication}

Using the VCCC, the EC and VSMC were loaded with Fluo-4 and en face measurements of changes in intracellular $\mathrm{Ca}^{2+}$ were recorded as previously described [7]. Briefly, the acetomethoxyester (membrane-permeable) form was dissolved in HBSS $(2.5$ $\mu \mathrm{mol} / \mathrm{l}$, with $0.25 \%$ pluronic $\mathrm{F} 127$ and $0.1 \% \mathrm{DMSO}, 25 \mathrm{mmol} / \mathrm{l}$ HEPES; Invitrogen), incubated for $20 \mathrm{~min}$ at $37^{\circ} \mathrm{C}$ and allowed to de-esterify in the HBSS for $20 \mathrm{~min}$ at $37^{\circ} \mathrm{C}$ and mounted on an Olympus FV200 confocal microscope. For measurements of changes in intracellular $\mathrm{Ca}^{2+}$ across the pores of the Transwells (where the in vitro MEJs are found [16]), pores were first identified in VSMC and 8 line scans in the Z-direction (1.5 $\mu \mathrm{m}$ apart) towards the EC were obtained. In each case, the VSMC were stimulated with $10 \mu \mathrm{M}$ phenylephrine (PE) applied from a micropipette $(0.5 \mathrm{~mm}$ tip) positioned $1 \mathrm{~mm}$ over the cell surface as a current pulse at $500 \mathrm{nA}$ and $1 \mathrm{~s}$ as previously described [24]. A background image of the Transwell devoid of cells was subtracted from the pixel intensity of each image $\left(\mathrm{F}_{\text {sub }}\right)$. Maximum fluorescence intensity $\left(\mathrm{F}_{\max }\right)$ of both cell types was determined at the termination of each experiment by application of $10 \mu \mathrm{M}$ ionomycin and stimulation with $100 \mu \mathrm{M}$ ATP. Relative $\left[\mathrm{Ca}^{2+}\right]_{i}$ values are plotted as the $\% \mathrm{~F}_{\max }$.

Phosphorylation at the MEJ
Inhibitors

Using the VCCC, the acetomethoxyester form of BAPTA (20 $\mu \mathrm{M}$; Invitrogen) [25] was selectively loaded into VSMC [7, 17] following loading of Fluo-4 for $20 \mathrm{~min}$ at room temperature, with 15 min allowed for de-esterfication. Xestospongin C (XPC, blocker of the $\mathrm{IP}_{3}$ receptor; $20 \mu \mathrm{M}$; Sigma) [26] selectively applied to EC $[7,17]$ was added to Fluo-4-loaded cells for $10 \mathrm{~min}$ at room temperature before experiments. Application of 18 GA (50 $\mu$ M; Sigma) was performed as described [7].

\section{Statistics}

A minimum of 3 mice were used for all in vivo experiments, and 3 separate cremasteric EC and VSMC isolations for all VCCC experiments. $\mathrm{p}<0.05$ determined by one-way ANOVA (Tukey post hoc test) was considered to be significant; error bars in figures are $\pm \mathrm{SD}$.

\section{Results}

In vivo Dye Transfer in Mouse Cremaster Arterioles Is Influenced by Connexin Phosphorylation

We have previously demonstrated that $\mathrm{Cx} 43$ is phosphorylated at S368 on actin bridges between EC and VSMC in mouse cremaster arterioles [8], and this particular phosphorylation residue is associated with a more closed state of gap junction [15]. Because cAMP is a potent enhancer of gap junctional intercellular communication (GJIC) [27-30], we explored whether activation of this pathway could increase GJIC from VSMC to EC. Application of the cAMP analog PCPT to mouse cremasters resulted in a significant decrease in the amount of S368 identified on the MEJ using transmission electron microscopy (fig. 1a, b) as well as actin bridges (fig. 1c, d). When antibody detection on the actin bridges was quantified, the GJIC blocker carbonoxolone did not have a direct effect on S368 phosphorylation (fig. 1e).

To observe how decreased detection of Cx43 S368 on actin bridges may correlate with altered functional GJIC, we microinjected a single VSMC and monitored dye transfer to neighboring cells. Consistent with published results in mouse cremasteric arterioles, there was no detectable dye transfer from the microinjected VSMC to neighboring ECs (fig. 2a, b) [14]. To test if loss of phosphorylation at $\mathrm{Cx} 43$ S368 could induce dye transfer, cremasters were treated with pCPT and a single VSMC was microinjected. Under these conditions, a significant increase in dye transfer was detected in neighboring ECs (fig. 2c-e). Further, dye transfer in the presence of pCPT was inhibited with carbonoxolone (fig. 2e). This data is consistent with pCPT-limiting phosphorylation at $\mathrm{Cx} 43$ S368 at the MEJ resulting in a gap junction channel more permeable to dye transfer. 
Cx43 S368 Correlates with Altered Dye Permeability in the Cremasteric VCCC

We used the cremasteric VCCC to look more closely at regulation of heterocellular communication by phosphorylation of $\mathrm{Cx} 43$ at S368. In control conditions, there was no detectable level of Cx43 S368 (fig. 3a). Application of PMA (fig. 3b) or FGF-2 (data not shown) resulted in extensive phosphorylation of $\mathrm{Cx} 43$ S368. In functional assays from VSMC to EC, both FGF-2 and PMA significantly reduced dye transfer when compared with control (fig. 4). These experiments are similar to that observed in vivo where functional GJIC between VSMC and EC is reduced when $\mathrm{Cx} 43$ is phosphorylated at $\mathrm{S} 368$ at the MEJ.

\section{Cx43 S368 Is Associated with Altered Calcium}

Communication in the Cremasteric VCCC

Although dye transfer is an accepted assay to monitor coupling between cells with open gap junctions, it does not always correlate with the transfer of physiologically significant molecules. We therefore sought to measure calcium fluxes between the VSMC and EC through the in vitro MEJ. As demonstrated in figure 5a, line scans of pores after stimulation of VSMC with PE revealed calcium communication in the Z-direction moving from VSMC to EC. Quantification of the experiments under control conditions showed a gradual increase in calcium across the in vitro MEJ (fig. $5 \mathrm{~b}$ ) which is diminished at the approximate location of EC and VSMC contact [16] when gap junction inhibitors are applied (fig. 5c).

We next studied how calcium was communicated from VSMC to EC following application of PMA or FGF2. Application of PMA or FGF-2 caused an inhibition of calcium communication from VSMC to EC (fig. 6a, c), similar to treatment with gap junction inhibitors $[7,17]$. However, using the Z-scanning method described in figure 5 to examine calcium communication across the in vitro MEJ, we found a transient increase in intercellular calcium in the EC when the VCCC was treated with PMA or FGF-2 (arrows in fig. 6b, d), providing correlative evidence of an incomplete block by $\mathrm{Cx} 43 \mathrm{~S} 368$ phosphorylation of either $\mathrm{Ca}^{2+}$ or $\mathrm{IP}_{3}$.

Using the cremasteric VCCC, we have previously demonstrated that $\mathrm{IP}_{3}$ and $\mathrm{Ca}^{2+}$ move from VSMC to EC [7, 17]. To decipher which second messenger may be involved in the transient increase in calcium observed across the MEJ, we first loaded VSMC with BAPTA to chelate calcium ions in the VSMC and, thus, prevent their movement to EC via GJIC after VSMC stimulation with PE (fig. 6e). Treatment of VSMC with BAPTA effectively
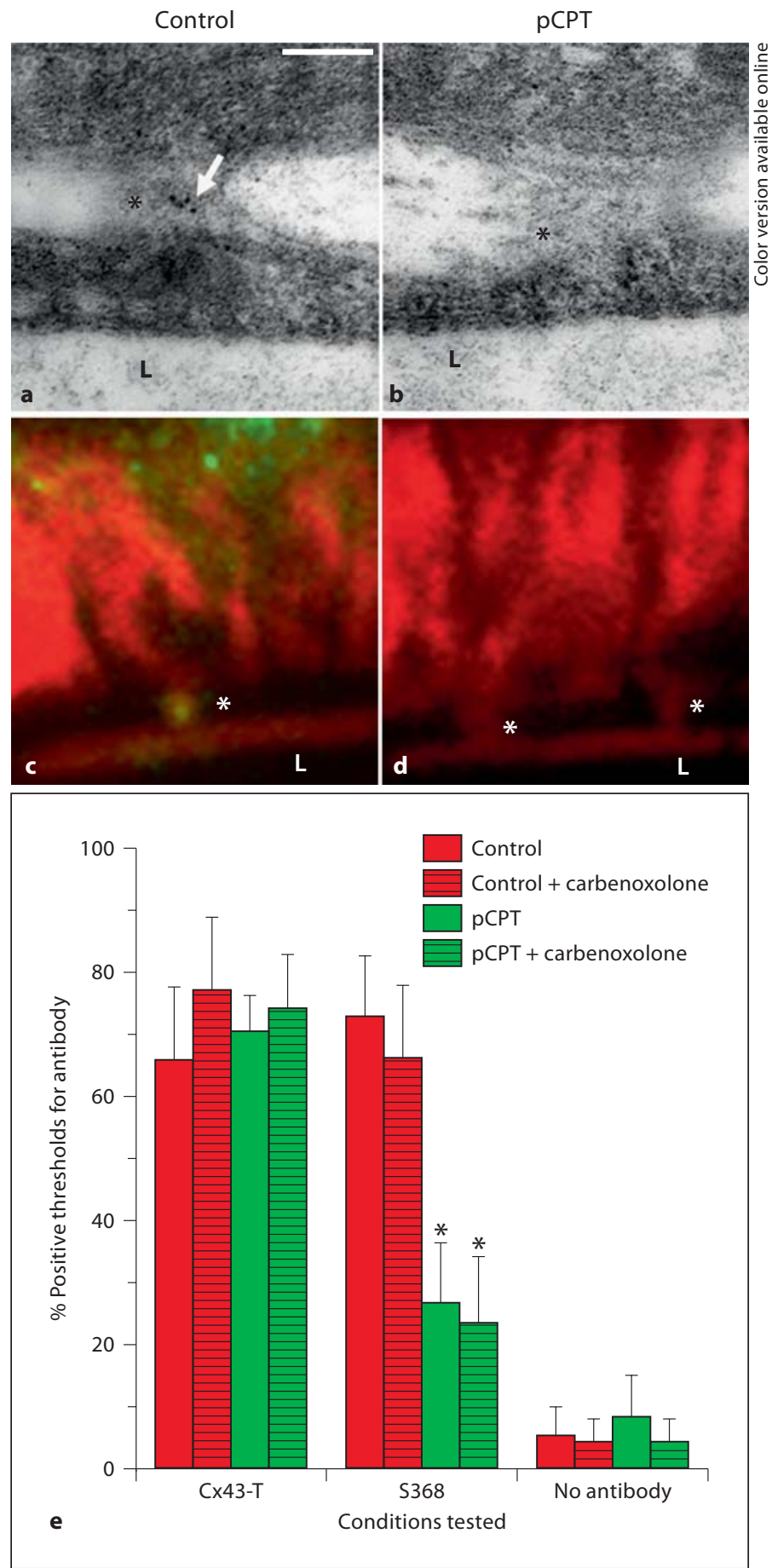

Fig. 1. Application of pCPT reduces phosphorylation of $\mathrm{Cx} 43$ at S368 on MEJs and actin bridges. Immunogold labeling $(\mathbf{a}, \mathbf{b})$ and actin bridge labeling (c, d) of S368 (green)/phalloidin (red) in control and pCPT-treated cremasteric arterioles. e Histogram of percent number of time that a protein was detected by antibodies on an actin bridge in mouse cremasteric arterioles. Bars are standard deviation. In $\mathbf{a}$, arrow indicates gold beads, in $\mathbf{a}-\mathbf{d}, \mathrm{L}$ indicates lumen of vessel and asterisks indicate MEJs. In e, asterisks represent a significant difference between control and treatment. 


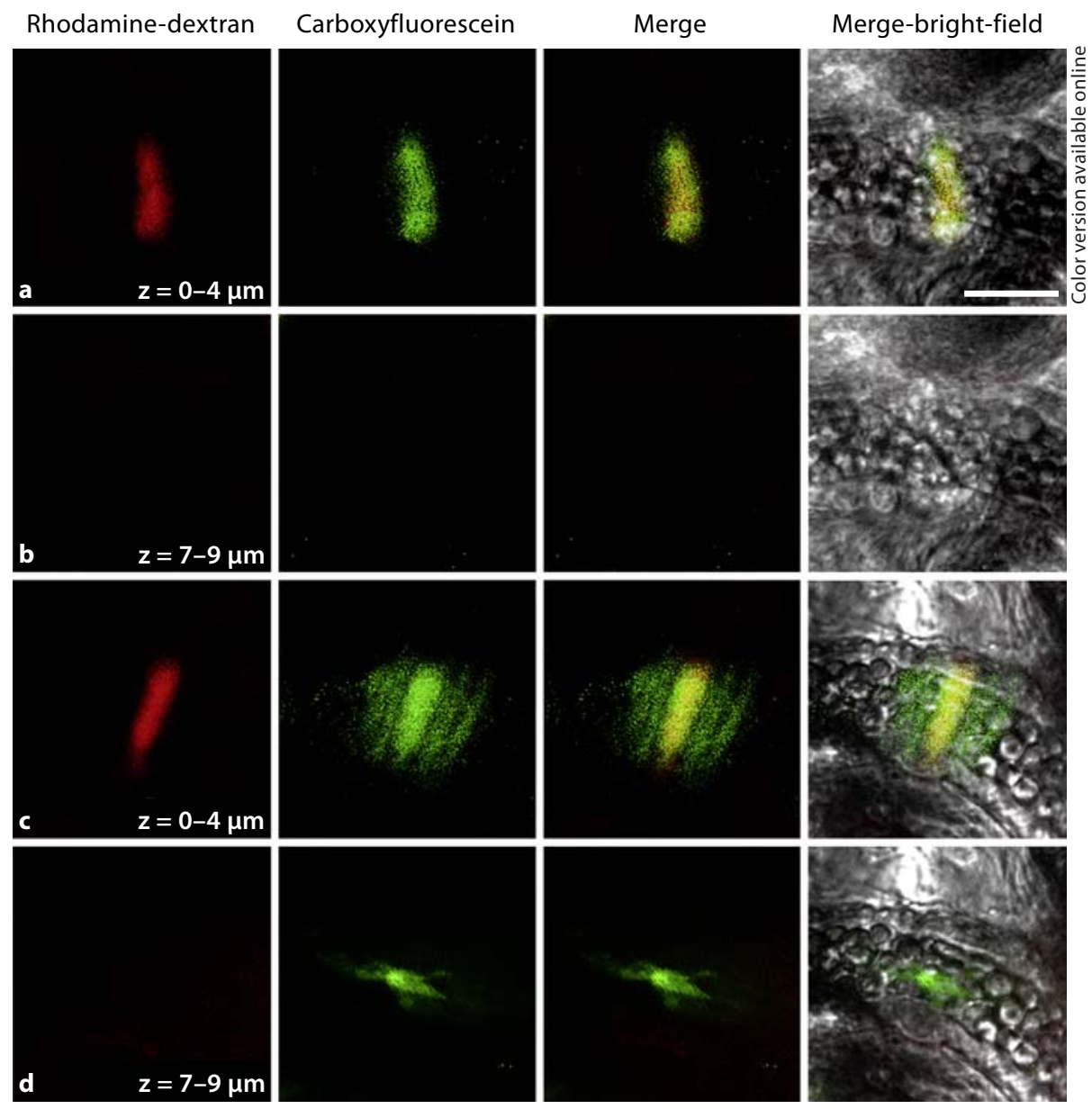

Fig. 2. In mouse cremaster arterioles, dye transfer from VSMC to EC is only observed after treatment with pCPT. In images a-d, raw data from single-cell microinjection of VSMC in control conditions $(\mathbf{a}, \mathbf{b})$ or conditions where pCPT was applied to the cremaster $(\mathbf{c}, \mathbf{d})$ are demonstrated. In a and $\mathbf{c}$, a single VSMC is seen after microinjection with rhodamine-dextran(red) and carboxyfluoresceine (green). At least 12 VSMC were injected per experimental paradigm in 3 separate mice. The 2 images are also seen merged and superimposed over the bright-field image of the vessel. In $\mathbf{b}$ and $\mathbf{d}$, a lower focal plane is used to observe EC. The scale bar in a is 30 $\mu \mathrm{m}$ and is representative for all images. Histogram in $\mathbf{e}$ is the percent incidence that the carboxyfluoresceine is observed in EC. * Significant difference between tested conditions.

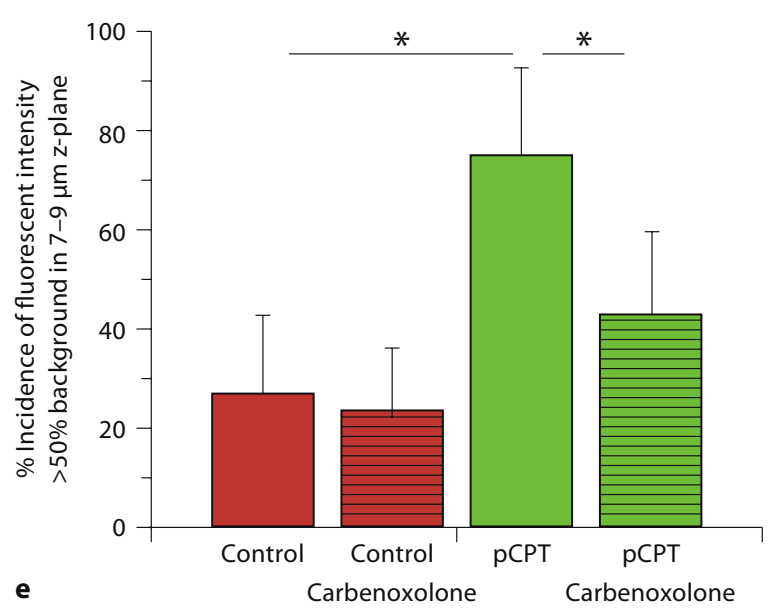



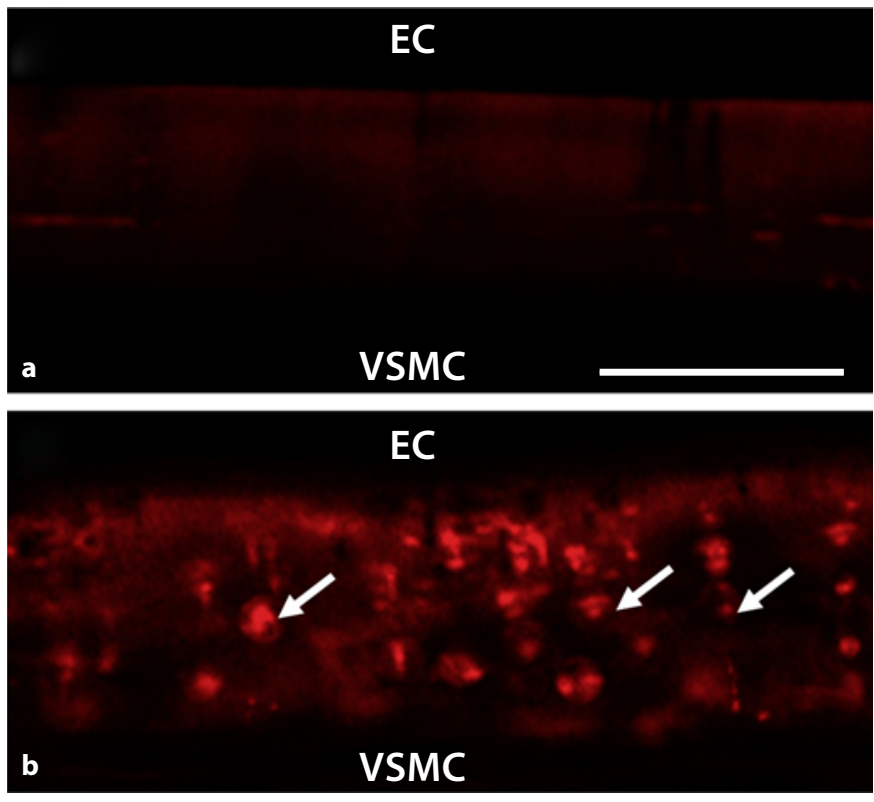

Fig. 3. Phosphorylation of $\mathrm{Cx} 43$ at $\mathrm{S} 368$ at the in vitro MEJ after application of PMA. Transverse view of VCCC stained for Cx43 S368 (red) in control (a) or after application of PMA (b); $\mathrm{n}=5$ different VCCC. Arrows indicate S368 labeling on the MEJ. The scale bar in $\mathbf{a}$ is $10 \mu \mathrm{m}$ and is representative for all images.

eliminated the calcium flux in EC, consistent with calcium movement through gap junctions. Alternatively, we loaded EC with XPC, a potent inhibitor of the $\mathrm{IP}_{3}-\mathrm{R}$, to prevent by $\mathrm{IP}_{3}$ derived from VSMC after VSMC stimulation with $\mathrm{PE}$ to cross the $\mathrm{MEJ}$ and raise intracellular calcium in the EC (fig. 6f). XPC had no effect on the calcium transient across the MEJ (fig. 6f). These data indicate that phosphorylation of $\mathrm{Cx} 43$ at S368 is correlated with inhibition of movement of $\mathrm{IP}_{3}$, and to a lesser extent the movement of $\mathrm{Ca}^{2+}$, across gap junctions from VSMC to EC at the MEJ.

\section{Discussion}

Although there is extensive paracrine signaling between EC and VSMC, the MEJ has also been shown to be an important signaling conduit for heterocellular communication in the vessel wall [for review, see 3]. The presence of gap junctions at the MEJ can allow for the movement of endothelium-derived hyperpolarizing factor (EC to VSMC) [31] or $\mathrm{IP}_{3}$ (VSMC to EC) [32] or possibly other small molecules that participate in direct cell-cell

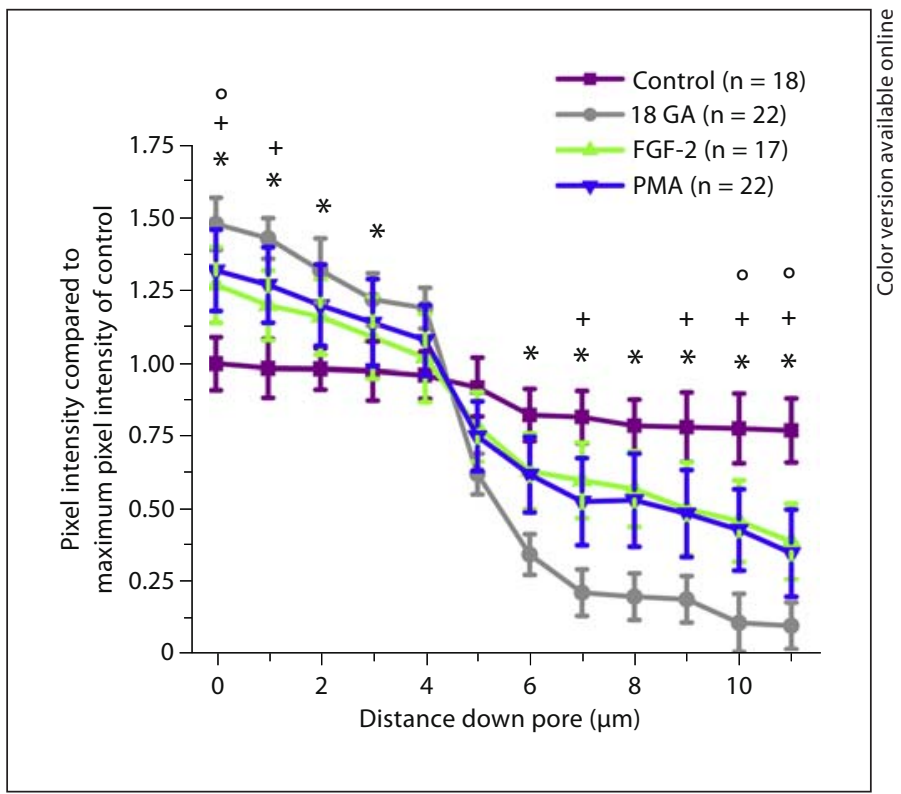

Fig. 4. Reduced dye transfer from smooth muscle to endothelium after treatment with FGF-2 or PMA. Biocytin was loaded into VSMC. Transfer of biocytin through the Transwell insert pores to EC at 1- $\mu \mathrm{m}$ intervals was determined by streptavidin pixel intensity. Four different experimental paradigms were tested; biocytin transfer from VSMC to EC (control, purple), biocytin transfer after application of $18 \mathrm{GA}$ (18 GA, gray), biocytin transfer after application of FGF-2 (FGF-2, green) and biocytin transfer after application of PMA (PMA, blue). $\mathrm{n}$ is the number of pores examined per paradigm. Symbols represent $\mathrm{p}<0.05$ when compared to control at each point along the pore length: * $18 \mathrm{GA} ;{ }^{\circ} \mathrm{FGF}-2$; + PMA.

signaling in the vasculature, but little is known about the mechanisms regulating the transfer of these small molecules through gap junctions. We have previously demonstrated that movement of $\mathrm{IP}_{3}$ from VSMC to EC is wholly dependent on the placement of $\mathrm{IP}_{3}-\mathrm{R} 1$ on the EC side of the MEJ [17]. However, these data did not indicate how gap junctions at the MEJ may (or may not) regulate solute movement. Using in vivo cremasteric arterioles and the VCCC, for the first time we provide key observations that confirm differential regulation of Cx43 S368 phosphorylation is associated with altered heterocellular communication at the MEJ. These data support the concept that the MEJ may be a highly regulated signaling domain in the vasculature and that the phosphorylation states of specific $\mathrm{Cx}$ residues can also help to mediate heterocellular signaling by regulating the open or closed state of gap junctions at this critical signaling structure. 


\section{Connexin Phosphorylation Regulation}

In previous work, we have shown an increased expression of phosphorylated Cx43 S368 on actin bridges in mouse cremaster arterioles in comparison with rat mesenteric arteries. Phosphorylation of the Cx43 S368 site has been demonstrated to be regulated by levels of PKC [11], as increases in PKC by phorbol esters result in an increased phosphorylation of S368 $[15,33]$. Here we show that by increasing cAMP using $\mathrm{pCPT}$, there is a decrease in the level of Cx43 S368 detected on actin bridges in mouse cremaster arterioles. The overlap of multiple signaling pathways in the regulation of Cx43 S368 are indicative of a highly regulated phosphorylation and dephosphorylation of this site that can contribute to intercellular signaling at the MEJ.

Work by Siegl et al. [14] observed a lack of heterocellular coupling between the EC and VSMC in mouse cremaster arterioles, noting significant differences in the response of electrical stimuli in each cell type and the poor or nonexistent dye transfer (carboxyfluoresceine). We confirmed this observation, showing that under control conditions, no dye transfer occurred from the VSMC to the EC. However, when we decreased Cx43 S368 phosphorylation, we were then capable of observing dye transfer from VSMC to EC, which was inhibited with carbonoxylone. This dye transfer pattern is similar to the that observed in rat mesentery and hamster cheek pouch [5, 13]. Our in vivo data therefore suggest that the regulation of $\mathrm{Cx} 43$ phosphorylation at the $\mathrm{S} 368$ residue could be an important component for controlling the transfer of solutes across the MEJ. However, there are several other phosphorylation sites on $\mathrm{Cx} 43$ (as well as on $\mathrm{Cx} 40$ and on $\mathrm{Cx} 37)$ that have not been examined directly in small molecule transfer studies in vitro or in vivo [34; for review, see 11]. Thus, we cannot rule out regulation of solute transfer through the modification of additional phosphorylation sites on $\mathrm{Cx} 43$ and/or by the regulation of additional Cxs present at the MEJ.

An alternative explanation for increased small molecule communication in response to PCPT used in these studies is a direct increase in the number of gap junctions at the MEJ, as cAMP has been shown to increase gap junction formation [30]. Under this hypothesis, gap junctions would not normally be present at the MEJ; however, pCPT application would induce movement of gap junctions to the MEJ and thus allow for heterocellular dye transfer. Although we cannot exclude this possibility, the time involved in translocation of the gap junction channels (for example, 1-5 h) limits this hypothesis under our experimental conditions.

Phosphorylation at the MEJ

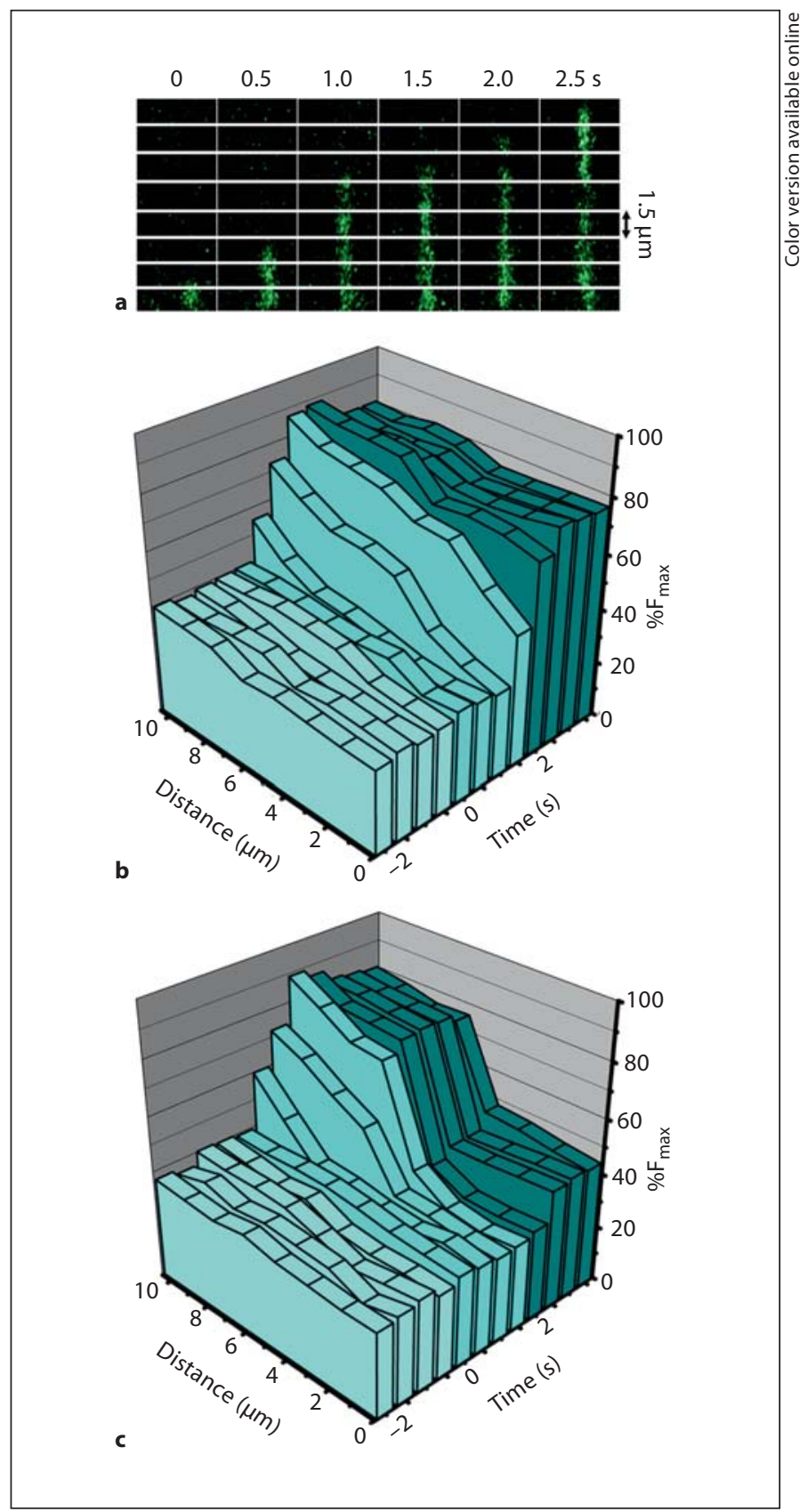

Fig. 5. Movement of an increase in intracellular calcium from VSMC to EC through the in vitro myoendothelial junction. a Line scans of a single pore from VSMC to EC over time with Fluo- 4 in green. $\mathbf{b}, \mathbf{c}$ The $\% \mathrm{~F}_{\max }$, time and distance through the pores of the Transwell are plotted in three-dimensional walls. In control conditions, there is a spread of an increase in calcium that moves from the VSMC to the EC through the pores (b). However, when the gap junction inhibitor 18 GA is applied to the cremasteric VCCC, the increase in calcium initiated in the VSMC is halted approximately half-way through the pore - the approximate location where EC and VSMC make contact [16] (c). Application of PE occurs at time point $0 \mathrm{~s}$; VSMC side of the pore is at distance $10 \mu \mathrm{m}$ and EC side of the pore is at distance $0 \mu \mathrm{m}$. 
Fig. 6. Phosphorylation of $\mathrm{Cx} 43$ at $\mathrm{S} 368$ inhibits $\mathrm{IP}_{3}$ flux from VSMC to EC. The VCCC was treated with PMA (a, b) or FGF-2 (c, d). In a and c, measurements of the intracellular calcium in the EC and VSMC monolayers (as previously described [7]) were compared to the calcium communication moving through the in vitro MEJ in the pores of the Transwell insert (b, d). With both treatments the red arrows indicate a transient increase in calcium on the EC side of the in vitro MEJ. When VSMC were additionally loaded with BAPTA-AM (e), the transient increase in calcium on the EC side of the in vitro $\mathrm{MEJ}$ is no longer present, while treatment of the EC monolayer with XPC failed to inhibit the transient increase in EC calcium (f).

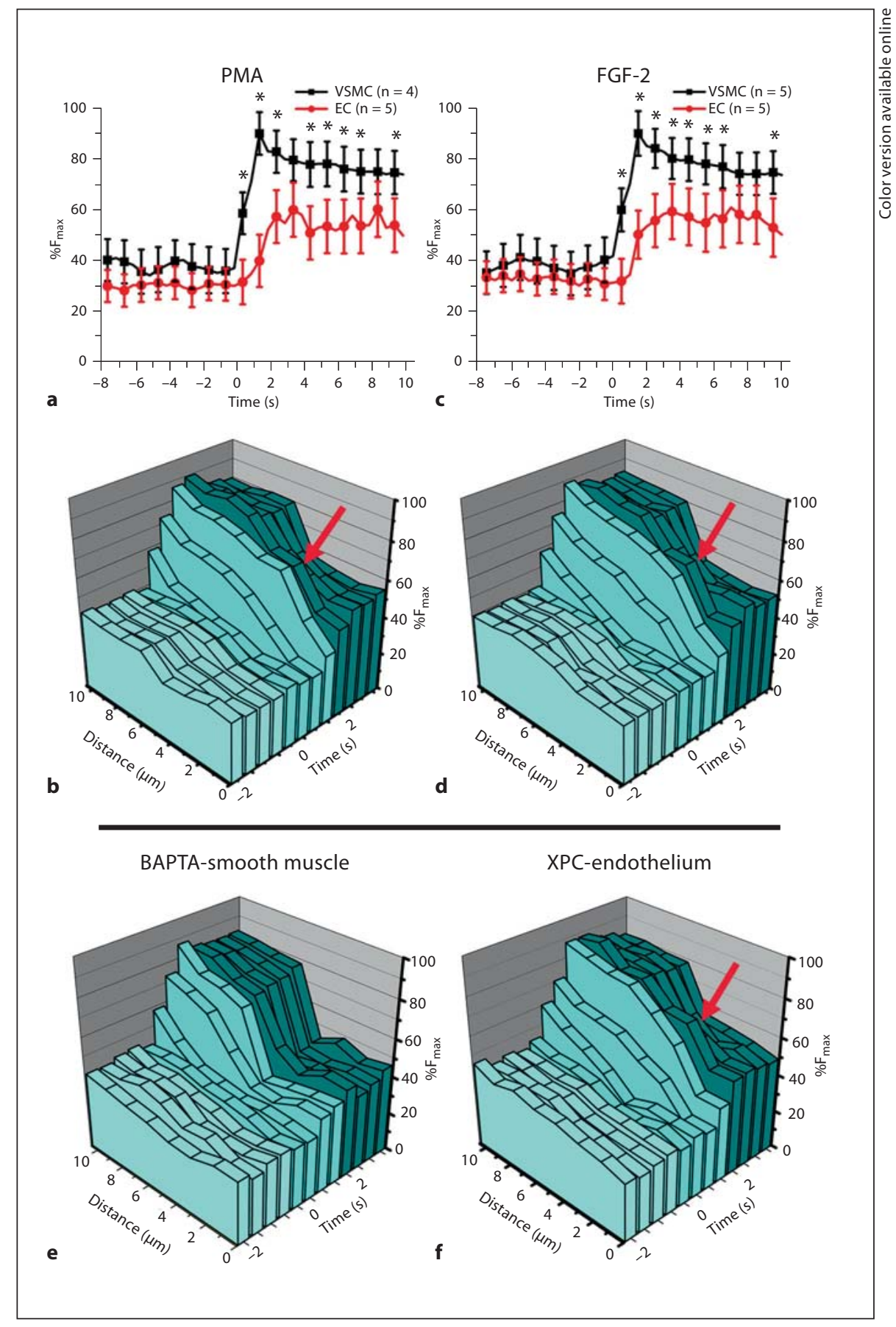

We further examined the effect of $\mathrm{Cx} 43$ phosphorylation in vitro using the VCCC. Although under control conditions we could not detect Cx43 S368 phosphorylation, using both PMA and FGF-2, we were able to induce phosphorylation at the in vitro MEJ. This phosphorylation correlated with a significant reduction in movement of biocytin from VSMC to EC down the length of the pores of the Transwell. These data correlate with in vivo cremasteric arterioles where dye transfer was not observed when Cx43 S368 was detected. In addition, because FGF-2 is an endogenous circulating molecule, our findings that it contributes to Cx43 S368 phosphorylation 
and regulation represents a potential in vivo target [35, 36]. This intriguing observation will require further in vivo evaluation.

Although dye transfer is a helpful indicator of functional gap junction communication, it does not always correlate with transfer of physiologically relevant solutes between cells. We therefore determined if the phosphorylation state of $\mathrm{Cx} 43 \mathrm{~S} 368$ could help to control the movement of $\mathrm{Ca}^{2+}$ and/or $\mathrm{IP}_{3}$ from VSMC to EC using the VCCC. Using the VCCC, we have previously demonstrated under normal conditions there was an observable transfer of $\mathrm{IP}_{3}$ and $\mathrm{Ca}^{2+}$ from VSMC to EC which was inhibited with gap junction inhibitors $[7,17]$. When we increased phosphorylation of the Cx43 S368 residue using either PMA or FGF-2, we observed an inhibition of intercellular communication, similar to when we treated the cells with gap junction uncouplers. Despite the overall inhibition, a higher level of intracellular calcium in EC remained after VSMC stimulation and we decided to further investigate this phenomenon by taking line scans of the Transwell pore so as to observe the increase in calcium movement from VSMC to EC across the in vitro MEJ. Under conditions that increased phosphorylation of $\mathrm{Cx} 43$ at S368, we observed a transient increase in $\mathrm{Ca}^{2+}$ in the EC. To accurately determine the nature of the second messenger inducing the transient increase in $\mathrm{Ca}^{2+}$, we used XPC to inhibit the activation of $\mathrm{IP}_{3}-\mathrm{R} 1$ in the EC monolayer, but still observed the increases in $\mathrm{EC}\left[\mathrm{Ca}^{2+}\right]_{\mathrm{i}}$. When we chelated $\mathrm{Ca}^{2+}$ in the VSMC layer with BAPTA, the $\mathrm{Ca}^{2+}$ transient in the EC was eliminated. Our data therefore are associated with $\mathrm{Cx} 43$ phosphorylation at S368 at the in vitro MEJ inhibits $\mathrm{IP}_{3}$ movement, but some $\mathrm{Ca}^{2+}$ still 'leaks' through the gap junction. These results strongly correlate the ability of phosphorylated $\mathrm{Cx} 43$ at the S368 residue to inhibit the transfer of physiologically relevant second messengers, in vitro.

Interestingly, $\mathrm{PKC}$ phosphorylation has been shown to decrease activity of $\mathrm{IP}_{3}-\mathrm{R} 1$, whereas PKA phosphorylation of the $\mathrm{IP}_{3}-\mathrm{R} 1$ has been shown to increase activity of the receptor [for review, see 37]. In these instances, the closure and opening of the gap junction channel would appear to act synergistically with the activity of the localized $\mathrm{IP}_{3}-\mathrm{R} 1$ at the MEJ [17] so as to regulate movement of second messengers between the EC and VSMC. It is possible then that posttranslational modifications acting in unison may be an important component to control of heterocellular communication.

A potential caveat with the VCCC experiments is the ability to phosphorylate $\mathrm{Cx} 43$ at S368 at levels consistent with those observed in vivo. Unfortunately, there is no direct comparison between the in vivo level of Cx43 S368 and the degree to which phosphorylation at S368 is artificially induced (using FGF-2 or PMA) in the VCCC. Therefore, the level of phosphorylation could significantly alter the flux of dye and/or second messengers between the 2 cell types.

\section{Why Are Cremaster Vascular Beds Different?}

Cremasteric skeletal muscle contributes to the movement of testis to provide for temperature regulation during physiological homeostasis. Our data have demonstrated that in the normal vessels, $\mathrm{Cx} 43$ is phosphorylated at Cx43 S368; however, this has not been observed in rat mesentery [8], indicating that this phosphorylation may be tissue specific. It is not clear why this occurs, but one possibility may be the temperature of the cremaster $\left(34^{\circ} \mathrm{C}\right)$ compared to the mesentery $\left(37^{\circ} \mathrm{C}\right)$; for example, the activities of $\mathrm{PKC}$ isoforms have been shown to be temperature sensitive [38]. In addition, it has been shown that in cremastric arteriole, $\mathrm{K}_{\mathrm{Ca}}$ channels have a high set point that renders them silent under resting conditions even in arterioles with substantial resting tone [39], which is in stark contrast to other studies demonstrating an estimated $\mathrm{K}_{\mathrm{Ca}}$ set point 6- to 18 -fold lower [40]. As mouse cremaster beds are used extensively in in vivo studies of microcirculation function, it is clear more research into this vascular bed-specific phenomena is required.

In conclusion, we have demonstrated for the first time that connexin phosphorylation at the MEJ may be an important regulatory mechanism for heterocellular communication from VSMC to EC both in vivo using the mouse cremaster arteriole and in vitro using the VCCC. We believe this may be an important method whereby heterocellular communication across the MEJ could be regulated.

\section{Acknowledgments}

We thank the University of Virginia Histology Core for sectioning of VCCC as well as the Advanced Microscopy Core for preparation of tissue for electron microscopy. This work was supported by NIH HL088554 (B.E.I.) and an American Heart Association Scientist Development Grant (B.E.I.). 


\section{References}

$\checkmark 1$ de Wit C, Boettcher M, Schmidt VJ: Signaling across myoendothelial gap junctions fact or fiction? Cell Commun Adhes 2008;15: 231-245.

$\checkmark 2$ Sandow SL, Haddock RE, Hill CE, Chadha PS, Kerr PM, Welsh DG, Plane F: What's where and why at a vascular myoendothelial microdomain signalling complex. Clin Exp Pharmacol Physiol 2009;36:67-76.

$\checkmark 3$ Heberlein K, Straub AC, Isakson BE: The myoendothelial junction: breaking through the matrix? Microcirculation 2009;16:307322 .

4 Rhodin JA: The ultrastructure of mammalian arterioles and precapillary sphincters. Ultrastruct Res 1967;18:181-223.

$\checkmark 5$ Mather S, Dora KA, Sandow SL, Winter P, Garland CJ: Rapid endothelial cell-selective loading of connexin 40 antibody blocks endothelium-derived hyperpolarizing factor dilation in rat small mesenteric arteries. Circ Res 2005;97:399-407.

-6 Dora KA, Sandow SL, Gallagher NT, Takano H, Rummery NM, Hill CE, Garland CJ: Myoendothelial gap junctions may provide the pathway for EDHF in mouse mesenteric artery. J Vasc Res 2003;40:480-490.

7 Isakson BE, Ramos SI, Duling BR: $\mathrm{Ca}^{2+}$ and inositol 1,4,5-trisphosphate-mediated signaling across the myoendothelial junction. Circ Res 2007;100:246-254.

$\checkmark 8$ Isakson BE, Best AK, Duling BR: Incidence of protein on actin bridges between endothelium and smooth muscle in arterioles demonstrates heterogeneous connexin expression and phosphorylation. Am J Physiol Heart Circ Physiol 2008;294:H2898-H2904.

-9 Haddock RE, Grayson TH, Brackenbury TD, Meaney KR, Neylon CB, Sandow SL, Hill CE: Endothelial coordination of cerebral vasomotion via myoendothelial gap junctions containing connexins 37 and 40. Am J Physiol Heart Circ Physiol 2006;291:H2047-H2056.

10 Sandow SL, Neylon CB, Chen MX, Garland CJ: Spatial separation of endothelial smalland intermediate-conductance calcium-activated potassium channels $(\mathrm{K}(\mathrm{Ca}))$ and connexins: possible relationship to vasodilator function? J Anat 2006;209:689-698.

$\checkmark 11$ Lampe PD, Lau AF: Regulation of gap junctions by phosphorylation of connexins. Arch Biochem Biophys 2000;384:205-215.

$\checkmark 12$ Lampe PD, Lau AF: The effects of connexin phosphorylation on gap junctional communication. Int J Biochem Cell Biol 2004;36: 1171-1186.

13 Little TL, Xia J, Duling BR: Dye tracers define differential endothelial and smooth muscle coupling patterns within the arteriolar wall. Circ Res 1995;76:498-504.

- 14 Siegl D, Koeppen M, Wolfle SE, Pohl U, de Wit C: Myoendothelial coupling is not prominent in arterioles within the mouse cremaster microcirculation in vivo. Circ Res 2005; 97:781-788
15 Lampe PD, TenBroek EM, Burt JM, Kurata WE, Johnson RG, Lau AF: Phosphorylation of connexin 43 on serine 368 by protein kinase $\mathrm{C}$ regulates gap junctional communication. J Cell Biol 2000;149:1503-1512.

16 Isakson BE, Duling BR: Heterocellular contact at the myoendothelial junction influences gap junction organization. Circ Res 2005;97:44-51.

17 Isakson BE: Localized expression of an Ins $(1,4,5) \mathrm{P} 3$ receptor at the myoendothelial junction selectively regulates heterocellular $\mathrm{Ca}^{2+}$ communication. J Cell Sci 2008;121: 3664-3673.

18 Isakson BE, Damon DN, Day KH, Liao Y, Duling BR: Connexin40 and connexin43 in mouse aortic endothelium: evidence for co ordinated regulation. Am J Physiol Heart Circ Physiol 2006;290:H1199-H1205.

19 Looft-Wilson RC, Payne GW, Segal SS: Connexin expression and conducted vasodilation along arteriolar endothelium in mouse skeletal muscle. J Appl Physiol 2004;97:1152-1158.

20 Cooper CD, Lampe PD: Casein kinase 1 regulates connexin-43 gap junction assembly. J Biol Chem 2002;277:44962-44968.

-21 Desaphy JF, De Luca A, Camerino DC: Blockade by cAMP of native sodium channels of adult rat skeletal muscle fibers. Am J Physiol 1998;275:C1465-C1472.

-22 Figueroa XF, Paul DL, Simon AM, Goodenough DA, Day KH, Damon DN, Duling BR: Central role of connexin40 in the propagation of electrically activated vasodilation in mouse cremasteric arterioles in vivo. Circ Res 2003;92:793-800.

23 Wiedeman MP: Blood flow through terminal arterial vessels after denervation of the bat wing. Circ Res 1968;22:83-89.

24 Langlands JM, Diamond J: The effect of phenylephrine on inositol 1,4,5-trisphosphate levels in vascular smooth muscle measured using a protein binding assay system. Biochem Biophys Res Commun 1990;173: 1258-1265.

25 Yashiro Y, Duling BR: Integrated $\mathrm{Ca}^{2+}$ signaling between smooth muscle and endothelium of resistance vessels. Circ Res 2000;87: 1048-1054.

26 Oka T, Sato K, Hori M, Ozaki H, Karaki H: Xestospongin C, a novel blocker of IP3 receptor, attenuates the increase in cytosolic calcium level and degranulation that is in duced by antigen in RBL-2H3 mast cells. $\mathrm{Br}$ J Pharmacol 2002;135:1959-1966.

27 Albright CD, Kuo J, Jeong S: cAMP enhances Cx43 gap junction formation and function and reverses choline deficiency apoptosis. Exp Mol Pathol 2001;71:34-39.

-28 Somekawa S, Fukuhara S, Nakaoka Y, Fujita H, Saito Y, Mochizuki N: Enhanced functional gap junction neoformation by protein kinase A-dependent and Epac-dependent signals downstream of cAMP in cardiac myocytes. Circ Res 2005;97:655-662.
29 Paulson AF, Lampe PD, Meyer RA, TenBroek E, Atkinson MM, Walseth TF, Johnson RG: Cyclic AMP and LDL trigger a rapid enhancement in gap junction assembly through a stimulation of connexin trafficking. J Cell Sci 2000;113:3037-3049.

30 Abudara V, Garces G, Saez JC: Cells of the carotid body express connexin 43 which is up-regulated by cAMP. Brain Res 1999;849: 25-33.

31 Dora KA, Gallagher NT, McNeish A, Garland CJ: Modulation of endothelial cell KCa3.1 channels during endothelium-derived hyperpolarizing factor signaling in mesenteric resistance arteries. Circ Res 2008; 102:1247-1255

32 Lamboley M, Pittet P, Koenigsberger M, Sauser R, Beny JL, Meister JJ: Evidence for signaling via gap junctions from smooth muscle to endothelial cells in rat mesenteric arteries: possible implication of a second messenger. Cell Calcium 2005;37:311-320.

33 Ek-Vitorin JF, King TJ, Heyman NS, Lampe PD, Burt JM: Selectivity of connexin 43 channels is regulated through protein kinase C-dependent phosphorylation. Circ Res 2006;98:1498-1505.

-34 Lampe PD, Cooper CD, King TJ, Burt JM: Analysis of Connexin43 phosphorylated at S325, S328 and S330 in normoxic and ischemic heart. J Cell Sci 2006;119:3435-3442.

35 Fujii T, Yonemitsu Y, Onimaru M, Inoue M, Hasegawa M, Kuwano H, Sueishi K: VEGF function for upregulation of endogenous PlGF expression during FGF-2-mediated therapeutic angiogenesis. Atherosclerosis 2008;200:51-57.

$36 \mathrm{Xu} \mathrm{H}$, Czerwinski P, Hortmann M, Sohn HY, Forstermann U, Li H: Protein kinase C alpha promotes angiogenic activity of human endothelial cells via induction of vascular endothelial growth factor. Cardiovasc Res 2008;78:349-355

37 Yule DI, Straub SV, Bruce JI: Modulation of $\mathrm{Ca}^{2+}$ oscillations by phosphorylation of Ins $(1,4,5) \mathrm{P} 3$ receptors. Biochem Soc Trans 2003;31:954-957.

38 Hansra G, Garcia-Paramio P, Prevostel C, Whelan RD, Bornancin F, Parker PJ: Multisite dephosphorylation and desensitization of conventional protein kinase $\mathrm{C}$ isotypes. Biochem J 1999;342:337-344.

39 Jackson WF, Blair KL: Characterization and function of $\mathrm{Ca}^{2+}$-activated $\mathrm{K}+$ channels in arteriolar muscle cells. Am J Physiol 1998; 274:H27-H34.

40 Benham CD, Bolton TB, Lang RJ, Takewaki T: Calcium-activated potassium channels in single smooth muscle cells of rabbit jejunum and guinea-pig mesenteric artery. J Physiol 1986;371:45-67. 